\title{
EVIDENCE OF SYSTEMATIC ERRORS IN FK5
}

L.V. Morrison (RGO), R.W. Argyle (RGO), Y. Requième (Bordeaux), L. Helmer (CUO), C. Fabricius (CUO), O.H. Einicke (CUO), M.E. Buontempo (RGO), J.L. Muiños (ROA), M. Rapaport (Bordeaux)

The primary purpose of the FK5 is to provide an absolute optical reference frame against which to measure the positions and motions of other objects. One of the main tasks of meridian circles is to check and improve that reference frame by repeated observation of the fundamental stars. The meridian circle at Bordeaux, France and the Carlsberg Automatic Meridian Circle (CAMC) at La Palma are carrying out programmes of observation of FK5 stars partly with this aim in view.

The positions from which the systematic differences are derived are the means of at least 10 , but normally about 30 (Bordeaux) and 50 (CAMC), independent observations of each FK5 star made in the period 1984-87. The systematic differences are displayed in pairs of 3-dimensional plots, Bordeaux-FK5 and CAMC-FK5. The similarities of the corresponding pairs of plots is striking, particularly in declination. This implies that systematic errors exist in the FK5 which reach about 0.1 in places at the epoch 1986.0.

A discussion of the results of other contemporary meridian circles and astrolabes at different latitudes is required in order to arrive at definitive corrections to the FK5. Perhaps this should be done before fitting the zero points of the Hipparcos catalogue to the FK5 system. 